\title{
Reconstructing Internal Temperature Distributions From Ultrasonic Time-of-Flight Tomography And Dimensional Resonance Measurements
}

\author{
S. J. Norton, L. R. Testardi, and H. N. G. Wadley \\ National Bureau of Standards, Washington, DC 20234
}

Accepted: November 7, 1983

\begin{abstract}
Two ultrasonic techniques for reconstructing the internal temperature distribution in metal bodies-timeof-flight tomography and dimensional resonance profiling-are described. An analysis of the tomographic reconstruction of temperature (including ray refraction effects) in a cylindrical body is presented together with initial experimental results. Dimensional resonance profiling is a new technique that allows the reconstruction of a one-dimensional distribution of temperature in a structure from measurements of its resonant frequencies. While time-of-flight tomography is well suited for measuring temperature in a cylindrical geometry, a combination of dimensional resonance and (a restricted form of tomography is the best method for measuring temperature profiles in the more practically important rectangular slab geometry.
\end{abstract}

Key words: dimensional resonance; metals processing; process control; temperature distribution sensor; tomography; ultrasound.

\section{Introduction}

The development of a temperature distribution sensor would be an important step in improving the productivity and quality of metals processing and reducing its energy needs. This has been recognized by the American Iron and Steel Institute [1] $]^{1}$, and a collaborative program of research with the National Bureau of Standards has been initiated to develop a sensor capable of providing internal temperature maps (to within $20^{\circ} \mathrm{C}$ ) with $20 \mathrm{~mm}$ spatial resolution or less for a variety of metals processing situations. Anticipated applications include measuring the internal temperature distribution in steel ingots during reheating and monitoring the temperature profile of steel strands as they are withdrawn from a continuous caster. Because of the limited time available for making the measurements, an ideal sensor should be capable of reconstructing temperature reliably with a

About the Authors: S. J. Norton, L. R. Testardi, and H. N. G. Wadley are with the Center for Materials Science in NBS' National Measurement Laboratory.

\footnotetext{
${ }^{1}$ Figures in brackets indicate references at the end of this paper.
}

minimum of measurements to avoid interfering with production processes.

We report here on two distinct but complementary ultrasonic techniques for reconstructing internal temperature in metals and other materials: time-offlight tomography and a new method which we call dimensional resonance profiling $[2,3]$. We identify the experimental and theoretical advantages and limitations of the two techniques for various geometries likely to be encountered in practice, and we propose a combination of the two exploiting their particular strengths for the most important practical geometry, the slab of rectangular cross-section.

The measurement of internal temperature by tomographic or dimensional resonance methods is based on the experimental observation that the velocity of sound in metals varies in a predictable way with temperature [4]. From room temperature to the melting point, the velocity of sound in austenitic steel and aluminum alloys decreases approximately linearly with temperature with a slope on the order of $-1 \mathrm{~m}$ $\mathrm{s}^{-1} /{ }^{\circ} \mathrm{C}$. A similar behavior is observed for ferritic steels, although it is somewhat complicated by the bcc to fcc phase change. In the temperature reconstructions reported below, we have assumed for simplicity a linear relation between temperature and velocity 
over the temperature range of interest. However, the use of a piece-wise linear relationship, with a change in slope over a higher range in temperature to approximate the bcc to fcc phase change, was found to introduce no serious complications during reconstruction.

\section{Time-of-Flight Tomography}

The measurement of the time-of-flight (TOF) of an ultrasonic pulse along a path penetrating a steel sample, for example, gives the line integral of the reciprocal sound velocity along that path. The path length divided by the TOF is also the average velocity along the path, and in fact could be used simply to compute the average temperature along that path. However, many TOF measurements over multiple intersecting paths, when employed as input to a tomographic algorithm, can be used to reconstruct a cross-sectional image of the sound velocity within the sample. Using the predetermined velocity-temperature relationship for the metal, the velocity map may then be converted into an image of internal temperature. In this section, we consider the application of tomography to two simple object geometries-cylindrical billets and rectangular slabs-representative of those found in metals processing.

\subsection{Cylindrical Billet}

We have, for simplicity, examined the TOF tomography problem for a cylindrical steel billet under the assumption that the temperature distribution is also cylindrically symmetrical. ${ }^{2}$ A crucial advantage of the assumption of circular symmetry is that the number of TOF measurements required for satisfactory spatial resolution is reduced perhaps two orders of magnitude below that of the general case (i.e., under no symmetry assumptions). For the symmetrical case, the unknown temperature reduces to a onedimensional function of radius. This simplification is important because a hostile measurement environment and time constraints impose severe limits on the

\footnotetext{
${ }^{2}$ When the shape of the billet is cylindrical and the boundary condition on the surface is symmetrical, this assumption is probably quite reasonable if the billet has been cooling for a short time. This is because heat-flow theory predicts that any initial asymmetrical spatial frequency components of the temperature damp out faster than the low-order symmetrical components [6]. As a result, the temperature tends rapidly toward a symmetrical distribution as the asymmetrical temperature gradients attenuate. (We assume throughout this paper that the thermal conductivity of the body is uniform.)
}

number of TOF measurements that can be conveniently and reliably performed during processing.

\subsubsection{Theory}

To measure temperature, our main task is to reconstruct the radial velocity profile $v(r)$ of the cylindrical billet. The velocity is then converted to a temperature profile $T(r)$ using, for example, a linear relationship between velocity and temperature of the form

$$
T(r)=T_{0}+b\left[v(r)-v_{0}\right],
$$

where $T_{0}, v_{0}$ and $b$ are experimentally-determined constants.

To recover the radial velocity distribution, a single "fan beam" measurement (i.e., paths radiating outward from one source and ending at an array of receivers) is sufficient. Let $\tau_{m}$ denote the measured TOF over a path $L_{m}$, as illustrated in figure 1. Suppose TOF measurements are obtained over $M$ distinct paths penetrating the cylinder. Then

$$
\tau_{m}=\int_{L m} \frac{d l}{v(r)}, \quad m=1,2, \ldots, M .
$$

The number $M$ will be small because of time constraints and the difficulty in making each measurement during processing. Since the angular range over which the TOF data are measured may be limited and sparsely sampled, an important factor in selecting a reconstruction algorithm is how well it performs with only limited data.

With a complete set of path-integral measurements $\tau_{m}$, a variety of tomographic algorithms could be used for recovering $v(r)$. Among these are the convolutionbackprojection, Fourier inversion, and algebraic reconstruction techniques (ART) [5]. Convolutionbackprojection, while computationally fast and well suited for commercial x-ray tomography, was not used here. This algorithm does not generally perform well when the data are limited in angle and/or are sparsely sampled; severe aliasing artifacts often result under these conditions. Fourier inversion is the Fourierdomain equivalent of convolution-backprojection and offers no advantages.

A second disadvantage of these "direct" approaches is that a priori constraints are not easily incorporated. This is undesirable because much a priori information is potentially available in the tomographic temperature problem, such as surface temperature, or a priori bounds on the range of the internal temperature distribution or the smoothness (gradient) of the 
Isotherms

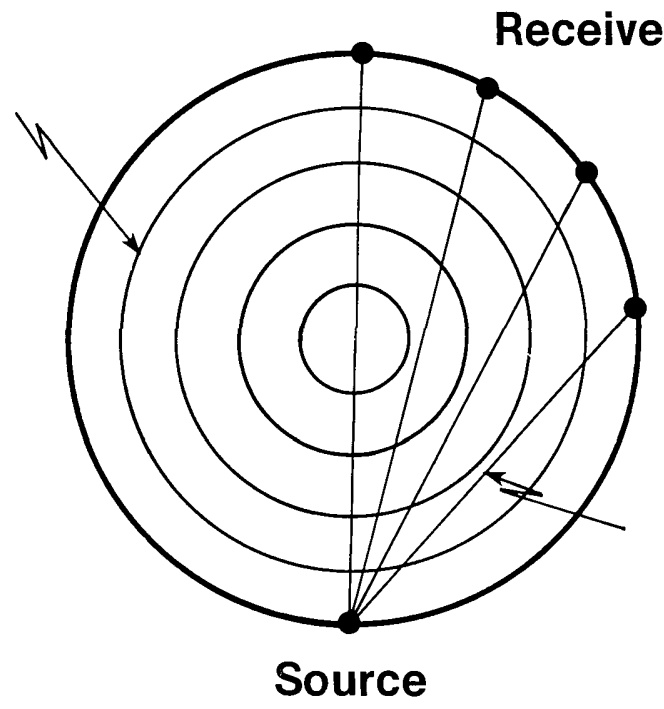

Figure 1-Cylinder cross-section with ray paths diverging from a single uitrasonic source. The temperature distribution is assumed to have cylindrical symmetry.

\section{Propagation path $\mathrm{L}_{\mathrm{m}}$}

temperature distribution. Another constraint is imposed by the thermal conductivity equation, which the temperature distribution must obey. Thus, reconstructions obtained at different times are not independent but are coupled by the heat flow equation. If one has a priori information on a temperature distribution at some initial time (e.g., that the temperature was initially uniform), then it might be possible to exploit this information to place bounds on the temperature or its gradient at subsequent times. We mention other beneficial effects of a priori constraints in a later section.

In contrast, the iterative ART algorithms are generally less susceptible to limited-data effects and allow incorporation of a priori information. We have found, however, that for the cylindrically-symmetric problem, for which the number of unknowns is relatively small, the complexity of an iterative technique is unnecessary.

For these reasons, none of the above algorithms is particularly well-suited for the simple cylindrical reconstruction problem. Instead, a "series expansion" algorithm was used and found to be both a natural approach in terms of imposing constraints (such as surface temperature, if known) and an effective way of reducing the number of unknown image pixels (or basis functions; see below) to an absolute minimum. The latter advantage is of fundamental importance because it implies a corresponding reduction in the number of measurements.

The series expansion technique consists of expanding the unknown profile (i.e., the reciprocal velocity) in a suitable set of basis functions, where "suitable" means that a truncated expansion (to, say, $N$ terms, where $N$ is small) provides a satisfactory approximation to the unknown profile. That is, we expand the reciprocal velocity using $N$ basis functions $\phi_{n}(r):$

$$
\frac{1}{v(r)}=\sum_{n=1}^{N} a_{n} \phi_{n}(r)
$$

where $\left\{\phi_{n}(r)\right\}$ is a basis set orthogonal on the interior of a circle of radius $R$ (the radius of the cylinder). We consider two choices of basis functions below, although, for an infinite basis set, any choice would suffice to expand $1 / v(r)$. However, as noted, the choice of $\left\{\phi_{n}\right\}$ will be dictated (in part) by how well a truncated series can be made to approximate $1 / v(r)$ for $0 \leqslant r \leqslant R$.

Inserting eq (3) into eq (2) and interchanging orders of summation and integration gives

$$
\tau_{m}=\sum_{n=1}^{N} a_{n} \Phi_{m n}, \quad m=1,2, \ldots, M
$$

where

$$
\Phi_{m n}=\int_{L m} \phi_{n}(r) d l
$$

Once the basis functions $\phi_{n}(r)$ are chosen, the matrix elements $\Phi_{m n}$ can be numerically computed and stored. Our problem then reduces to solving the linear system eq (4) for the unknown coefficients $a_{n}$, where the $\tau_{m}$ are measured. Upon solving eq (4) for $a_{n}$, eq (3) gives the reconstruction of the reciprocal velocity $1 / v(r)$, which in turn can be substituted into eq (1) to obtain 
the temperature profile $T(r)$. Generally, to mitigate the effect of measurement errors, many more TOF measurements $(M)$ than unknowns $(N)$ are desirable. In this case, eq (4) will be over-determined, and it is natural to compute the pseudoinverse (the minimumnorm least-mean-square-error solution) of eq (4). To illustrate this, write eq (4) in matrix form:

$$
\boldsymbol{\tau}=\Phi \mathbf{a}
$$

where $\Phi$ is an $M$ by $N$ matrix $(M \geqslant N)$, a is the $N$-component coefficient vector and $\tau$ is the $M$-component measurement vector. Minimizing the mean-square-error $E=\mathrm{e}^{T} \mathrm{e}$, where $\mathrm{e}=\Phi \mathbf{a}-\boldsymbol{\tau}$, results in the pseudoinverse of eq (6), given by

$$
\hat{\mathbf{a}}=\left(\Phi^{T} \Phi\right)^{-1} \Phi^{T} \boldsymbol{\tau},
$$

where ${ }^{T}$ denotes transpose.

In our work, two candidate basis sets $\left\{\phi_{n}\right\}$, Bessel functions and "ring functions," were studied. Both are orthogonal in the sense that

$$
\int_{0}^{R} \phi_{n}(r) \phi_{m}(r) r d r=N_{n} \delta_{n m}
$$

where $N_{n}$ is a normalization constant. They are defined as follows:

1) Bessel function basis:

$$
\phi_{n}(r)=J_{0}\left(k_{n} r\right)
$$

where $J_{0}(\cdot)$ is the zero-order Bessel function and $k_{n}$ is the $n$-th root of $J_{0}(k R)=0$.

2) Ring function basis:

$$
\phi_{n}(r)=\operatorname{ring}_{n}(r)
$$

where

$$
\begin{array}{r}
\operatorname{ring}_{n}(r)= \begin{cases}1 & \text { for } r_{n-1}<r \leqslant r_{n} \\
0 & \text { otherwise },\end{cases} \\
\text { and } r_{n}=R n / N, n=0,1, \ldots, N .
\end{array}
$$

The Bessel basis is a particularly interesting choice because Bessel functions are smooth over the circular domain, and we recall that the solution to the thermal conductivity equation in a cylindrical geometry is also given by a Bessel function series [6]. This suggests that the Bessel basis is a natural choice for the temperature reconstruction problem with circular symmetry and that the approximation (3) may even provide a good fit when truncated after the first few terms. This is because the higher-order terms in the series solution to the conductivity equation are exponentially damped with time. As a result, after a relatively short cooling time, the temperature profile increasingly resembles a single Bessel function, in which case only one term in eq (3) may be sufficient to approximate the profile.

The ring basis on the other hand provides a discrete or "staircase" approximation to the temperature profile, and thus does not provide the characteristic smooth temperature profile expected.

\subsubsection{Simulations and Experiments}

In our initial examination of the tomography problem, we performed computer simulations of temperature reconstructions. The following procedure was used: As a first step, we compute a hypothetical temperature profile by solving the thermal conductivity equation for a cooling cylinder. We assume in the simulation a thermal conductivity of 304 stainless steel, a 6-in-diameter cylinder, and an initially uniform temperature of $400^{\circ} \mathrm{C}$. Using the velocity-temperature relation, eq (1), we convert the radial temperature distribution into its corresponding velocity distribution. Given this hypothetical velocity profile, simulated TOF measurements are generated by numerically integrating the reciprocal velocity along $M$ propagation paths. These simulated TOF values are then used to compute $M$ values of $a_{n}$ by direct inversion of eq (6), i.e.,

$$
\mathbf{a}=\Phi^{-1} \boldsymbol{\tau}
$$

In this case, the number of measurements $\tau_{m}$ equals the number of unknowns $a_{n}$, and since $\Phi$ is full rank, direct inversion is possible. The resulting a is inserted into eq (3) to obtain the reconstructed velocity profile. The temperature reconstruction is then obtained by substituting the velocity into eq (1). When the Bessel function basis was used, the computed temperature profile was found to be almost indistinguishable from the original temperature distribution for cooling times 
longer than several hundred seconds. ${ }^{3}$ The values of $M$ used usually needed to be no larger than 2 or 3 for a good fit, thus confirming our earlier expectation that the Bessel basis is excellent for fitting temperature profiles, at least for an idealized cooling cylinder and after moderate cooling times.

Unfortunately, the matrix inverse $\Phi^{-1}$ is illconditioned, and on introducing random errors into $\tau_{m}$ on the order of a few tenths of a microsecond (corresponding to a 1:200 relative error), significant fluctuations in the calculated temperature profile $\left( \pm 50^{\circ} \mathrm{C}\right)$ resulted. The ill-conditioning problem became more dramatically apparent when real TOF measurements were obtained from a 6-in-diameter stainless steel cylinder. This cylinder was initially heated to $400^{\circ} \mathrm{C}$ and TOF measurements were generated by exciting $25 \mathrm{~ns}$ duration elastic wave pulses with a focused $30 \mathrm{~mJ}$ pulsed Nd:YAG laser. The received pulses were recorded with a PZT transducer coupled to the cylinder with a fused quartz buffer rod [7].

Eleven TOF measurements were made at increasing angles away from the cylinder diameter (fig. 1). Because of the limits on the accuracy of the measurements in this initial experiment, an attempt at a direct inversion using eq (9) failed for $M>2$. When more measurements were used, thereby increasing the order of the matrix $\Phi$, the problem becomes increasingly ill-conditioned. ${ }^{4}$ To verify that the reconstructed velocity distribution was consistent with the measured TOF values, we computed numerically the TOF values over each ray path using the reconstructed velocity distribution. The computed TOF values agreed with the measured TOF values to within $0.001 \mu \mathrm{s}$ or better, thereby confirming that the errors are not of a numerical origin, but arise due to uncertainty in the measurements themselves. Sources of uncertainty include the finite precision in measuring the TOF (about $\pm 0.05 \mu \mathrm{s}$ ), as well as other sources of error, such as variable grain anisotropy which affects wave propagation in the steel.

\footnotetext{
${ }^{3}$ The ratio between the coefficients multiplying the second and first Bessel functions in the series expansion of the heat-flow solution is $\exp \left(-14.7 \alpha t / R^{2}\right)$, where $\alpha$ is the thermal diffusivity of the metal and $R$ is the cylinder radius. This number gives us an indication of how fast the second- and higher-order terms attenuate with time relative to the first term, and thus how soon the first term will dominate. In our case, $\alpha / R^{2}=6.4 \times 10^{-4} \mathrm{~s}^{-1}$; letting, for example, $t=200 \mathrm{~s}$, the above ratio is 0.15 .

${ }^{4}$ A singular value decomposition of $\Phi$ shows condition numbers ranging from 3 to $10^{5}$ as the number of unknowns varies from 2 to 11 and depending on the choice of basis functions. The condition number provides an upper bound on the amplification of errors in the inversion process.
}

Another possible source of error is ray bending due to refraction. We show in the Appendix one method of estimating the error in a TOF measurement contributed by ray refraction. For the small temperature gradients encountered in our initial experiment $\left(\simeq 50^{\circ} \mathrm{C} / \mathrm{cm}\right)$, we found that the TOF errors due to refraction were quite negligible. Longer propagation paths and higher temperature gradients could, however, make ray bending effects significant, in which case some compensation for refraction would be needed for accurate reconstructions. Refraction effects in ultrasonic tomography have been discussed in the literature, and a first-order correction to the TOF due to refraction has been reported [8]. Iterative correction approaches have also been proposed [9].

It was also found that the ring basis set generates a better conditioned matrix $\Phi$ than the Bessel basis set. Thus, in some sense, the ring functions constitute a more "linearly-independent" set than the Bessel functions, and as a consequence, the measurement errors are generally amplified by a greater factor for the Bessel set than for the ring set. Although the Bessel basis provides a smoother fit, the ring basis evidently has the advantage of improving the numerical conditioning of the inversion problem.

As a next step, the pseudoinverse eq (7) was used to compute a smaller number of unknowns $(N)$ than measurements $(M)$. Using all 11 measurements and solving for far fewer coefficients reduced the illconditioning to some extent, but error amplification still precluded solving for more than three coefficients.

Figures $2 \mathrm{a}$ and $2 \mathrm{~b}$ show reconstructed temperature profiles using the pseudoinverse, eq (7), and all 11 measurements. The squares indicate measured values of the internal temperature $\left( \pm 2{ }^{\circ} \mathrm{C}\right.$ accuracy) obtained with a thermocouple probe. ${ }^{5}$ In figure $2 \mathrm{a}$, a Bessel function basis set was used in which the series expansion [eq (3)] was truncated at two terms. In figure $2 b$, the ring basis set was used, again with only two terms retained. An attempt to use more than two or three terms resulted in a poorer approximation to the true temperature profile.

We suggest two fundamental approaches to reducing the degree of error amplification in the inversion problem. Increasing the precision of the TOF measurement is the first requirement. Faster digitization rates, reduced noise and greater receiver bandwidth should all contribute to an improvement in

\footnotetext{
${ }^{5}$ The noticeable difference between the temperature at the surface and at the four interior points is probably a consequence of the short cooling time of the cylinder (a few minutes), as well as the failure to heat the cylinder uniformly prior to the measurements.
} 


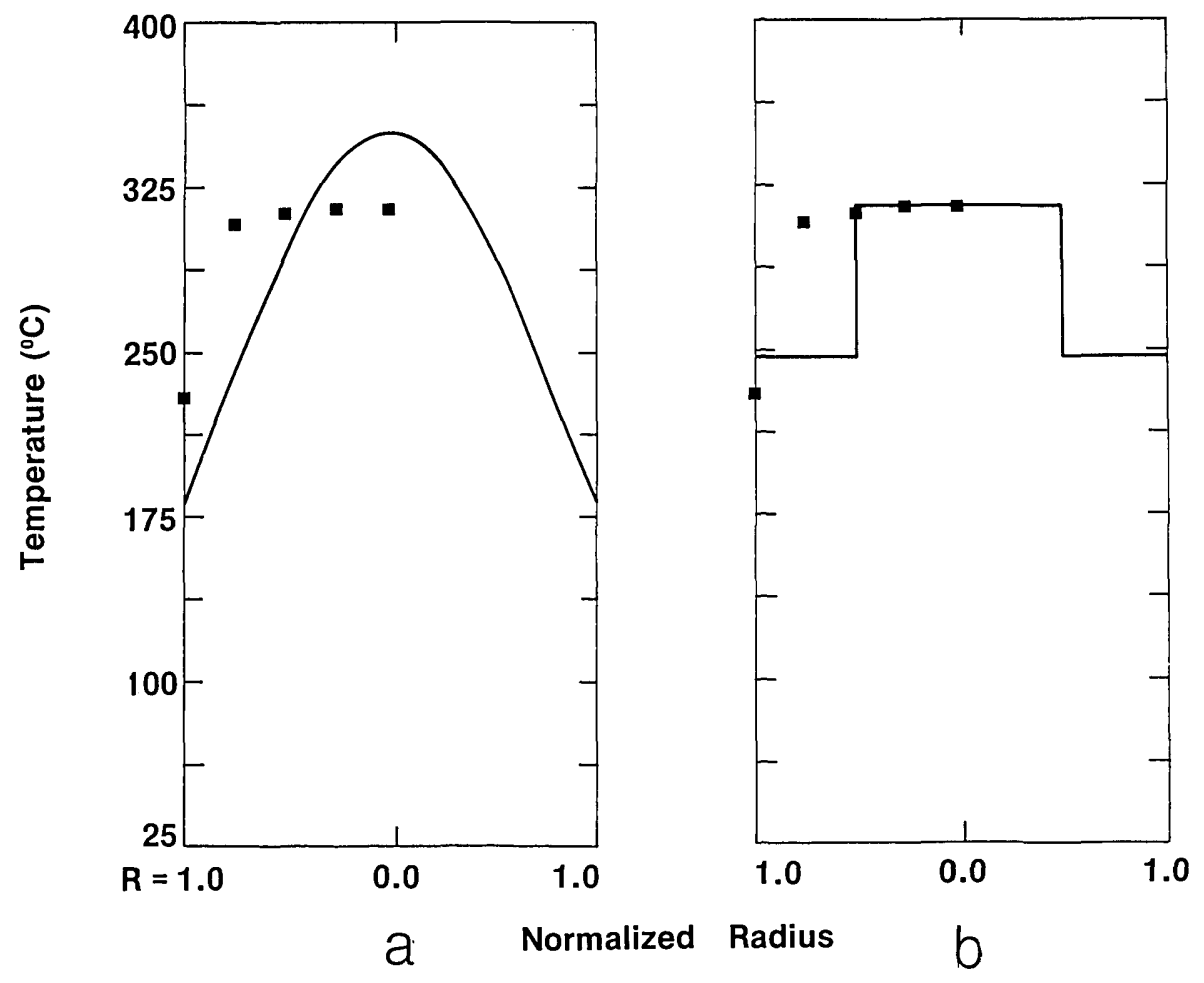

Figure 2-(a) Two-term Bessel function reconstruction of the temperature profile. The squares indicate the measured temperature. (b) Two-term ring function reconstruction of the temperature profile. the temporal resolution in the TOF measurement. We estimate that a minimum relative accuracy in the TOF measurement of approximately one part in $10^{3}$ is needed for acceptable accuracy $\left( \pm 20^{\circ} \mathrm{C}\right)$ and resolution $(\leqslant 20 \mathrm{~mm})$ in the tomographic reconstruction. For the 6-in cylinder used in the initial experiment, this represents an error of about $30 \mathrm{~ns}$ in the TOF. This TOF error corresponds to a pathlength error of $0.15 \mathrm{~mm}$, and so path lengths must also be measured to this sensitivity. Since the relative TOF error decreases in proportion to the length of the path, the longer path lengths expected in larger structures will help ease this exacting path-length precision.

The second approach to reducing error amplification is to use regularization or other numerically-stabilizing methods to reduce ill-conditioning. One such strategy is to impose a priori constraints on the solution. An important example of this is to constrain the boundary value to match the surface temperature, provided the surface temperature can be measured. Simulations demonstrate that such a surface constraint is effective in improving numerical stability. Another approach is to minimize the mean square error subject to a smoothing constraint on the velocity profile, which can be regarded as a form of regularization. A singular value decomposition of the matrix $\Phi$, combined with a judicious elimination of the smallest singular values, is also a potentially effective way of improving the numerical conditioning of the inversion problem [10].

Ill-conditioning of this kind is not peculiar to the series-expansion approach to tomography; in fact, error amplification is characteristic of all inverse problems. In general, when more terms in the series expansion (i.e., more unknowns) are retained, the more ill-conditioned the problem becomes, and the factor by which errors are magnified rapidly increases. This illustrates a fundamental trade-off between spatial resolution and the attainable accuracy in the reconstructed temperature. For a given uncertainty in the TOF measurements, only a finite number of unknowns (i.e., terms in the series) can be reliably computed. An attempt to compute more than this results in an error amplification so large as to seriously degrade the accuracy of the reconstructed temperature.

\subsection{Rectangular Slab}

In this section, we examine the possibility of using TOF tomography to reconstruct the internal temperature of a slab with rectangular cross-section (fig. 3). If the slab thickness is small relative to its height and breadth, the temperature gradient will be predominantly normal to its larger surface (i.e., the isotherms in fig. 3 will run parallel to the $x-y$ plane 


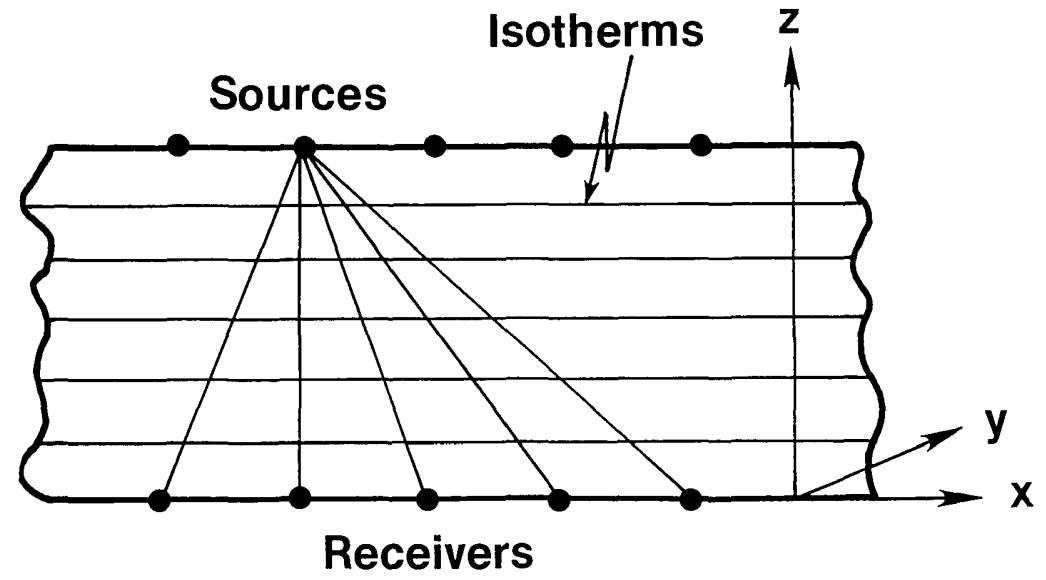

Figure 3-Slab cross-section with sources and receivers on opposite surfaces. The isotherms are assumed parallel to the surface.

with the gradient pointing in the $z$-direction). To a first approximation, it is reasonable in this case to regard the temperature as a function of $z$ only, with negligible variation in the $x$ and $y$ directions. This approximation again represents a major simplification in the reconstruction problem, because the unknown temperature profile is now only one-dimensional.

Unfortunately, if the isotherms run truly parallel to the edges, as assumed, a tomographic scheme will be ineffective if the sources and receivers are constrained ${ }^{6}$ to lie on either side (fig. 3). Line integrals over different paths intersecting the parallel layers sample the various layers in precisely the same proportion; the resulting system of equations is consequently linearly dependent, and any attempted inversion to recover the different velocities in the parallel layers will fail.

This problem is fundamental, and no tomographic algorithm, whether analytical or iterative, will succeed here. An equivalent interpretation of the "noninvertibility" of the measurements is this: With the sources and receivers on opposite sides, no propagation path lies parallel to the slab surface. In reconstruction-from-projections theory, this condition defines a so-called "limited-angle problem," and the well-known central slice theorem [5] predicts that the spatial frequency component corresponding to variations in the slab in the $z$-direction cannot be recovered from the limited-angle measurements. In particular, propagation along paths parallel to the slab surface are required to retrieve this component.

On the other hand, a limited form of tomography may be used if we restrict the possible temperature profiles to a particular form or shape. If this shape has

\footnotetext{
${ }^{6}$ This statement also applies if the sources and receivers lie on one side of the slab and the paths are defined by reflecting the pulses from the opposite surface.
}

one undetermined parameter, one TOF measurement will suffice to compute it. As an example, suppose we assume a symmetrical profile with the shape of a half sine wave. That is, if the slab thickness is $L$, we assume a temperature profile of the form

$$
T(z)=T(0)[1+a \cdot \sin (\pi z / L)], 0 \leqslant z \leqslant L,
$$

where $T(0)=T(L)$ is the surface temperature, which, for simplicity, we assume can be measured. The undetermined parameter, $a$, can then be recovered with a single TOF measurement; we see that the center temperature $(z=L / 2)$ is $T(0)(1+a)$.

Finally, an alternative, or complement, to this approach is the method of dimensional resonance profiling which is capable of recovering the spatial variations in temperature in the $z$-direction. This is described next.

\section{Dimensional Resonance Profiling}

This technique represents a novel approach to reconstructing a one-dimensional inhomogeneity in the elastic modulus and density of a body from measurements of its resonant frequencies [2,3]. An analysis reveals a simple relation between the coefficients of a Fourier expansion of the modulus and density and the measured values of the fundamental and overtone frequencies. The theory as developed thus far treats only the one-dimensional problem, and experiments successfully demonstrating the technique have been performed to date on a thin brass rod. For the case of a slab, the dimensional resonance approach should in principle allow one to reconstruct (from thickness resonance frequencies) the thickness variations of the modulus and density, from which the 
velocity of sound may then be computed. The thickness velocity profile can then be converted to temperature in the usual way. The theory of dimensional resonance is outlined below; a more complete treatment may be found in $[2,3]$.

\subsection{Theory}

Consider a one-dimensional object of length $L$ along the $z$-axis, where $0 \leqslant z \leqslant L$. Assume stress-free boundary conditions, and that the variations in the modulus and density are small relative to their dc components. Then the $n$-th order normal mode (dimensional resonance) is approximately

$$
u(z, t)=\sqrt{ } 2 / L \cos (n \pi z / L) \cos \left(\omega_{n} t\right),
$$

where $u(z, t)$ denotes displacement and $\omega_{n}$ is the (perturbed) resonance frequency. Represent the spatial variations of the linear elastic modulus by the Fourier series

$$
M^{L}(z)=M_{0}^{L}+\sum_{m=1}^{\infty} M_{m}^{L} \cos (m \pi z / L), \quad 0 \leqslant z \leqslant L
$$

and the linear density $\rho^{L}(z)$ by a similar series (the superscript $L$ stands for "linear," meaning $M^{L}$ and $\rho^{L}$ have units of modulus and density per unit length). If the acoustic damping is small, we can equate the timeaveraged kinetic energy (KE) and potential energy (PE) of the structure:

$$
\mathrm{KE}=\int_{0}^{L} \frac{1}{2} \rho^{L} \overline{\dot{u}^{2}} \mathrm{~d} z=\mathrm{PE}=\int_{0}^{L} \frac{1}{2} M^{L} \overline{\epsilon^{2}} \mathrm{~d} z
$$

where $\epsilon=\partial u / \partial z$ is the strain and the over-bar denotes time average. Substituting eqs (10) and (11) into eq (12) gives to first order in small quantities

$$
\frac{1}{2}\left(\frac{M_{2 n}^{L}}{M_{0}^{L}}+\frac{\rho_{2 n}^{L}}{\rho_{0}^{L}}\right)=\frac{\bar{\omega}_{n}{ }^{2}-\omega_{n}{ }^{2}}{\bar{\omega}_{n}{ }^{2}}
$$

where $\bar{\omega}_{n}=(n \pi / L)\left(M_{0}^{L} / \rho_{0}^{L}\right)^{1 / 2}$ are the resonant frequencies associated with the dc values of the modulus and density, $M_{0}^{L}$ and $\rho_{0}^{L}$.

Equation (13) equates the $2 n$-th Fourier coefficient of the modulus and density to the shift in the resonant frequency of the $n$-th mode. We see from eq (13) that by measuring the shifts in the resonant frequencies, we obtain the even-order coefficients of the Fourier expansion of the inhomogeneity in modulus and density.

One limitation of the dimensional resonance technique is that only the even-order Fourier coefficients are recoverable; i.e., the anti-symmetric part of the variation is not measurable from the resonant frequencies alone. A second limitation is that the dc values of the modulus, $M_{0}^{L}$, and density, $\rho_{0}^{L}$, are not directly measurable from eq (13). However, the dc modulus under certain conditions may be obtained from a single TOF measurement along the $z$-direction (provided dispersion is properly accounted for), and the dc density can be estimated from the mass of the object $[2,3]$.

The first limitation is potentially the more serious of the two. However, when it is reasonable to assume that the temperature distribution is symmetrical about the center, the dimensional resonance approach is potentially effective in recovering the internal temperature profile through the short dimension of a slab. Dimensional resonance also has the advantage of employing relatively low-frequency standing waves, and is thus less susceptible to signal-to-noise loss arising from high ultrasonic attenuation in hot steel with large grain size. This technique is also exceedingly well-behaved numerically compared to the inherent ill-conditioning of two-dimensional tomography.

\subsection{Experimental Result}

Figure 4 shows the reconstructed temperature distribution along a brass rod (36 in long with a diameter of 0.25 in) heated near its center. In this experiment, extensional waves were excited and detected in the rod using a noncontact, electromagnetic (eddy-current) transducer at one end; a phasedlocked loop was used to measure the resonant frequencies to a precision of several parts in $10^{5}$. A more detailed description of this experiment can be found in [3].

The reconstruction in figure 4 was performed using the five lowest dimensional resonances. Because the actual temperature distribution was relatively smooth, the calculated profile from only five terms in the Fourier series agreed with the true temperature profile to within $5 \%$ at all points along the rod.

\section{Discussion}

We have seen that time-of-flight tomography represents one approach to reconstructing the radial temperature profile in a cylindrical body. Dimensional resonance, as thus far studied, is a one-dimensional 
Figure 4-Dimensional resonance reconstruction of ed near its center. the temperature distribution along a rod heat.

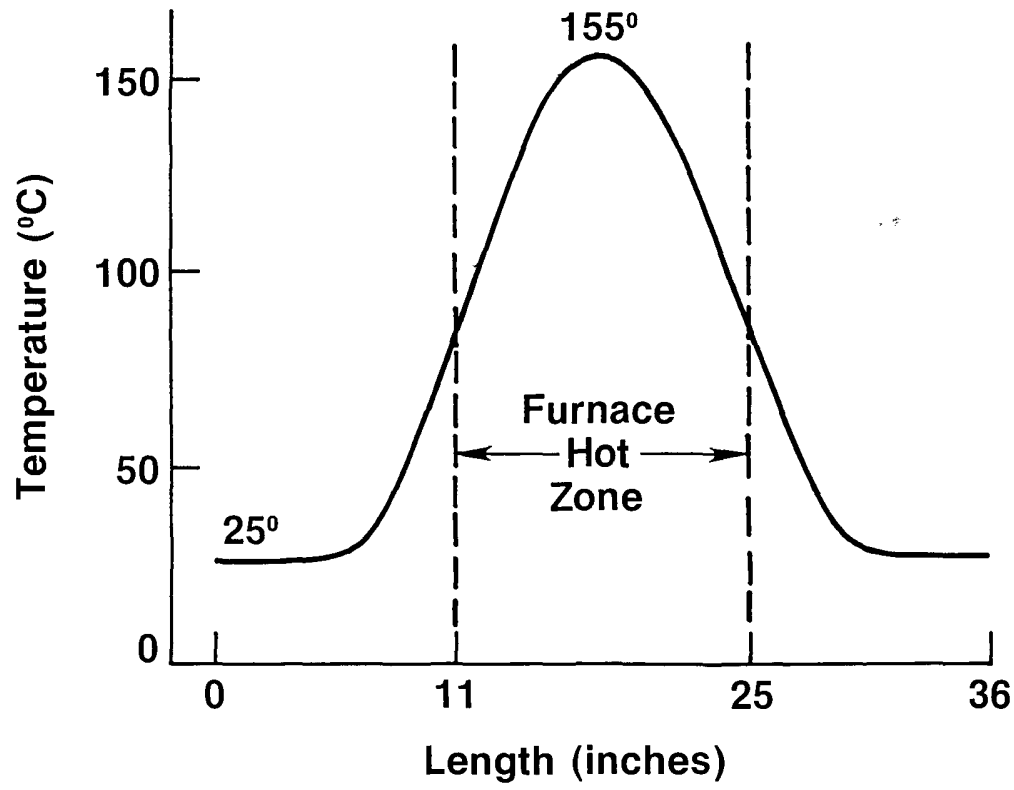

velocity gradient in the $y$-direction:

$$
v(y)=v_{0}+y v_{y},
$$

where $v_{y}=\partial v / \partial y$ is the velocity gradient and $v_{0}$ is the (constant) velocity along the $x$-axis. Let the source and receiver lie on the $x$-axis separated by the distance $l$ (fig. 5). In the absence of refraction $\left(v_{y}=0\right)$, the pulse will propagate along the $x$-axis with $\mathrm{TOF}_{0}=l / v_{0}$.

For the linear velocity gradient, given by eq (A1), the refracted path can be shown to be the arc of a circle intersecting the source and receive points. Defining $D$ as the radius of the circle and $\theta_{0}$ as the angle between the $x$-axis and the tangent to the circle at the source point (fig. 5), the ray path is given by

$$
\begin{aligned}
& y(\theta)=D \cos \theta-D \cos \theta_{0} \\
& x(\theta)=D \sin \theta+D \sin \theta_{0},
\end{aligned}
$$

for $-\theta_{0} \leqslant \theta \leqslant \theta_{0}$. Snell's law, for the linear gradient eq (A1), may be written

$$
v_{0} \cos \theta=v \cos \theta_{0}
$$
estimate of the center temperature is all that is needed.

\section{Appendix}

To estimate the error in a TOF measurement due to ray refraction, consider the simple example of a linear
Substituting eq (A1) into eq (A4) and the resulting expression for $\cos \theta$ into eq (A2), gives the relation

$$
v_{0}=D v_{y} \cos \theta_{0} .
$$




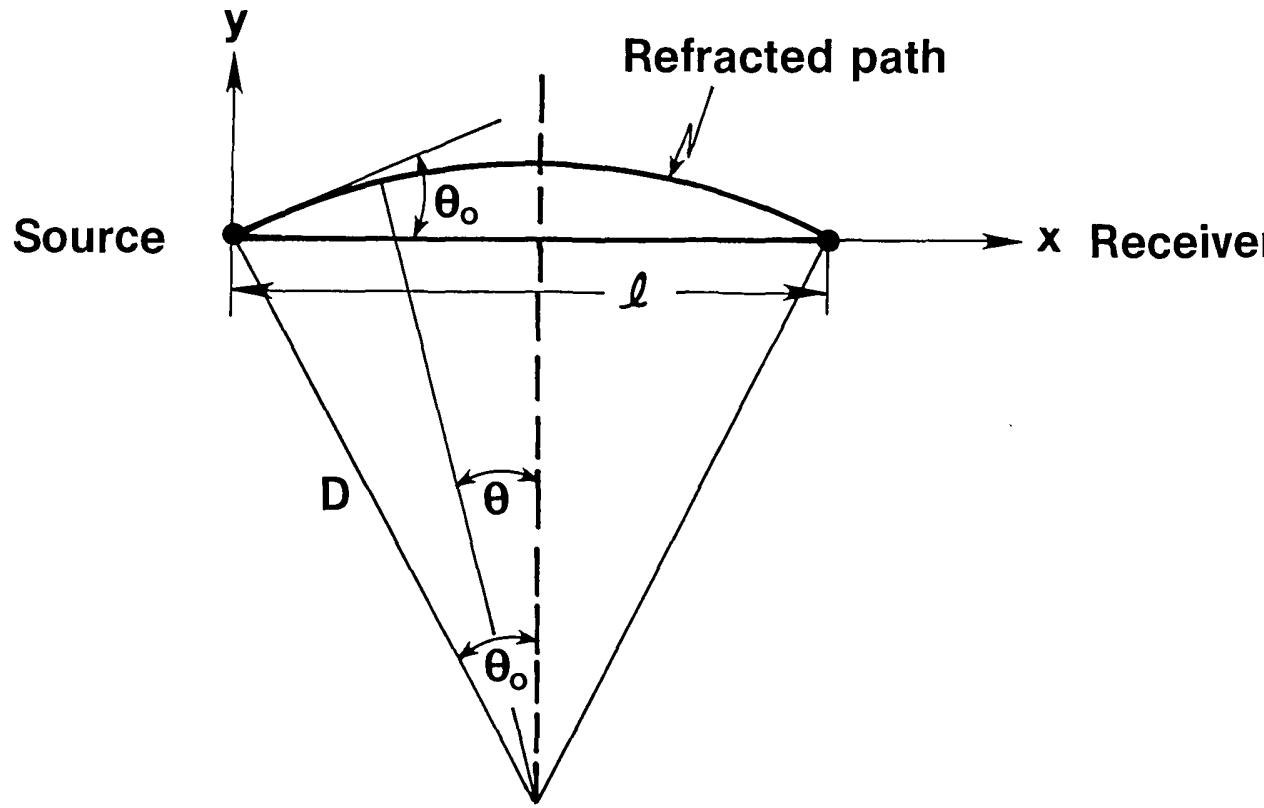

Figure 5-Refracted ray produced by a linear velocity gradient increasing in the $y$-direction.

From figure 5, we also have

$$
l=2 D \sin \theta_{0}
$$

The TOF over the refracted path is

$$
\mathrm{TOF}_{R}=\int_{-\theta_{0}}^{\theta_{0}} \frac{D d \theta}{v(\theta)}
$$

From eqs (A4) and (A5), we have $v(\theta)=D v_{y} \cos \theta$, so that

$$
\begin{aligned}
\mathrm{TOF}_{R} & =\frac{1}{v_{y}} \int_{-\theta_{0}}^{\theta_{0}} \frac{d \theta}{\cos \theta}=\frac{1}{v_{y}} \ln \left[\frac{1+\sin \theta_{0}}{1-\sin \theta_{0}}\right] \\
& =\frac{1}{v_{y}} \ln \left[\frac{1+l / 2 D}{1-l / 2 D}\right]
\end{aligned}
$$

Squaring and adding eqs (A5) and (A6) results in

$$
D=\left[(l / 2)^{2}+\left(v_{0} / v_{y}\right)^{2}\right]^{1 / 2}
$$

Assuming $D>>l$, we can insert eqs (A8) into (A7) and expand the result to first order in $l / D$, giving

$$
\mathrm{TOF}_{R}=\mathrm{TOF}_{0}\left[1-\frac{1}{24}\left(v_{y} l / v_{0}\right)^{2}\right]
$$

The second term on the right is the relative TOF error due to refraction (to first order in $l / D$ ).

\section{References}

[1] Report of Workshop on Process Control Sensors for the Steel Industry edited by Mehrabian, R., Whitely, R. L., van Reuth, E. C. and Wadley, H. N. G., Natl. Bur. Stand. NBSIR 82-2618 (1982).

[2] Norton, S. J.; Testardi, L. R. Reconstruction of onedimensional inhomogeneities in elastic modulus and density using dimensional resonances, J. Acoust. Soc. Am., to be submitted.

[3] Testardi, L. R.; Norton, S. J.; Hsieh, T. Determination of inhomogeneities of elastic modulus and density for onedimensional structures using acoustic dimensional resonances. J. Appl. Phys., to be submitted.

[4] Kurz, W.; Luz, B. Localization of the solid liquid interface in metals by ultrasonic methods. Z. Metallkunde 57: 70-73; Jan. 1966.

[5] Special Issue on Physical and Computational Aspects of 3-Dimensional Image Reconstruction. IEEE Trans. Nuclear Science NS-21; 1974.

[6] Carslaw, H. S.; Jaeger, J. C. Conduction of Heat in Solids, 2d Edition (Oxford Univ. Press, 1959).

[7] Wadley, H. N. G.; Norton, S. J.; Biancaniello, F. S.; Mehrabian, R. Ultrasonic Measurement of Internal Temperature Distribution. Proceedings of the 1983 American Society for Metals Congress, Philadelphia.

[8] Norton, S. J.; Linzer, M. Correcting for ray refraction in velocity and attenuation tomography: a perturbation approach. Ultrasonic Imaging 4: 201-233; 1982.

[9] Schomberg, H. An improved approach to reconstructive ultrasound tomography. J. Phys. D: Appl. Phys. 11: L181-L185; 1978.

[10] Forsythe, G. E.; Malcolm, M. A.; Moler, C. B. Computer Methods for Mathematical Computations (Prentice-Hall, Englewood Cliffs, NJ, 1977). 